\title{
Numerical Simulation of Wave Slamming on a Flap-Type Oscillating Wave Energy Device
}

\author{
P. J. Martínez Ferrer, Ling Qian*, D.M. Causon, C. G. Mingham, and Z. H. Ma Centre for \\ Mathematical Modelling and Flow Analysis, Manchester Metropolitan University \\ Manchester, United Kingdom
}

\begin{abstract}
This paper presents the numerical investigations of an oscillating wave surge converter (OWSC) operating in extreme sea states leading to slamming. We use the open-source computational fluid dynamics (CFD) library OpenFOAM to carry out the two-dimensional numerical simulations. A preliminary study is done to verify the convergence of our results, while scalability tests confirm the high-performance computing capabilities of OpenFOAM and the possibility of extending this study to large three-dimensional configurations. The OWSC device is simulated with both incompressible and compressible solvers, and the results are compared against previous numerical and experimental results. It is shown that an incompressible solver can capture the dynamics and general behavior of the flap device. Nevertheless, the compressibility effects can be reproduced only with the aid of a compressible solver, which takes into account the density changes in the air and water phases. Those effects produce high-frequency, small oscillations on the seaward side of the flap but do not contribute to further increasing the peak pressure values characteristic of slamming.
\end{abstract}

\section{INTRODUCTION}

The use of renewable energies, such as wind and solar, has experienced a noticeable increase in recent years. However, other sources of renewable energies, such as the one extracted from ocean waves nearshore, still remain largely underexploited at present. Therefore, more experiments and accurate numerical simulations need to be carried out in this area with special focus on structure survival as a consequence of harsh ocean conditions.

This paper focuses on the Oyster oscillating wave surge converter (Whittaker and Folley, 2012), which consists of a flap device hinged on the seabed and driven back and forth by the action of waves. The energy taken from the waves is utilized to pump fresh water into a hydraulic plant inshore, where it is finally converted into electricity. OWSCs obtain their maximum efficiency in nearshore locations of shallow water depths, where they acquire larger motions from the waves. One of the current challenges of the OWSCs is their survivability in extreme sea states, e.g., winter sea storms (Kay, 2014), in which large and infrequent extreme waves may compromise their structural integrity and consequently lead to an increase in their maintenance costs. Furthermore, global warming and climate change are likely to increase the frequency as well as the intensity of storms and hurricanes, which may influence the design of future OWSCs.

Several experiments and simulations have already been conducted to measure the loads on OWSCs under harsh ocean conditions, typically low tidal levels and large amplitude waves. The experimental scale model tests performed under those conditions indicated the presence of discrete slamming events (Henry et al., $2014 b$ ), which were identified as significantly large pressure peaks below and above the ambient pressure on the seaward wall surface

\footnotetext{
*ISOPE Member.
}

Received November 1, 2016; updated and further revised manuscript received by the editors April 24, 2017. The original version (prior to the final updated and revised manuscript) was presented at the Twentysixth International Ocean and Polar Engineering Conference (ISOPE2016), Rhodes, Greece, June 26-July 1, 2016.

KEY WORDS: Wave energy, oscillating wave surge converter, compressibility, slamming, impact, HPC simulation. of the OWSC. On the other hand, Henry et al. (2014a) carried out two-dimensional numerical simulations of the same experiment using the incompressible commercial CFD software Ansys Fluent. Although the incompressible numerical simulations seemed to capture well the general behavior and dynamics of the flap device, the authors could not predict any slamming events. In their simulations, they attributed the absence of large peak pressures produced by slamming to the presence of air entrapment and associated compressibility effects (Henry et al., 2014a), which were ignored by the incompressibility assumptions.

During violent wave impacts and slamming events, the fluid compressibility can play a major role in the correct prediction of impact loadings (Lugni et al., 2010). Aeration effects may also become important in the presence of enclosed air bubbles and air pockets trapped in water bodies (Bullock et al., 2007). Furthermore, the effect of body rotation (Xu et al., 2010) is of special importance in the particular case of an oscillating device. Therefore, the commonly incompressible assumption of the fluid, i.e., the air and water mixture, retained in previous simulations of OWSCs may yield to a numerical underestimation of the maximum impact loadings on OWSCs (Henry et al., 2014a).

In this paper, a battery of simulations is conducted on the basis of two-dimensional experiments on the slamming of an OWSC. Firstly, we describe briefly the numerical methods of the incompressible and compressible models used. Secondly, we analyze the convergence of our numerical results. Then we perform scalability tests to assess the performance of these simulations in a High Performing Computing (HPC) environment. Finally, we carry out a new numerical simulation of the OWSC utilising a compressible Navier-Stokes solver and compare our results against the experiments and incompressible data in order to determine whether the compressibility effects become important during slamming. In this regard, power take-off systems of the OWSC were not considered.

\section{NUMERICAL PROCEDURES}

\section{Incompressible and Compressible Navier-Stokes Solvers}

The numerical procedures considered in this paper rely on a cell-centered, co-located, finite-volume method already implemented in the open-source CFD library OpenFOAM (Jasak, 


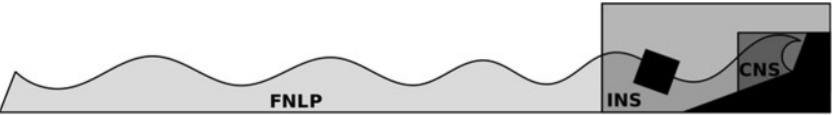

Fig. 1 Virtual Wave Structure Interaction (WSI) simulation environment

1996). The source code of this library is freely available and thus can be modified and further improved, which facilitates the use of third-party software and the development of in-house applications. For this reason, the adoption of OpenFOAM is increasing in both the research and industry. We have developed a new OpenFOAM-based solver, wsiFoam, for the study of the wave interaction between structures and floating bodies. The aim of wsiFoam is to gather specialized solvers, e.g., Fully Nonlinear Potential (FNLP), Incompressible Navier-Stokes (INS), and Compressible Navier-Stokes (CNS) solvers, at strategic locations of the computational domain and couple them through special interfaces in order to obtain the most efficient and accurate description of the underlying physical characteristics of a numerical wave tank (see Fig. 1).

For the simulations carried out in this work, we utilize the incompressible and compressible Navier-Stokes solvers integrated in wsiFoam. These two solvers are an extension of interFoam and compressiblelnterFoam, respectively. A detailed description of these two pressure-based solvers and wsiFoam can be found in Martínez Ferrer et al. (2016). We solve the three-dimensional Navier-Stokes equations applied to a two-phase flow by using the volume of fluid (VOF) method with additional terms to maintain a sharp interface between the phases (interface capturing) (see Rusche, 2002 and Weller, 2002). On the one hand, the incompressible solver assumes constant densities of the air and water phases, which simplifies the treatment of the mass-transport equation and the derived pressure-correction equation. On the other hand, the compressible model considers the air and water phases as a function of both the pressure and temperature. This requires solving additional equations to account for the density and temperature variations. The importance of these additional terms in the evaluation of the compressibility effects and the correct prediction of loadings during wave impacts has been already assessed by $\mathrm{Ma}$ et al. (2014) and Martínez Ferrer et al. (2016). To the authors' best knowledge, a compressible solver has not been previously employed to study the slamming phenomena of an OWSC.

\section{Numerical Setup}

Figure 2 illustrates the computational domain near the OWSC used to carry out the simulations. The hybrid mesh combining tetrahedral and triangular cells was generated by the open-source

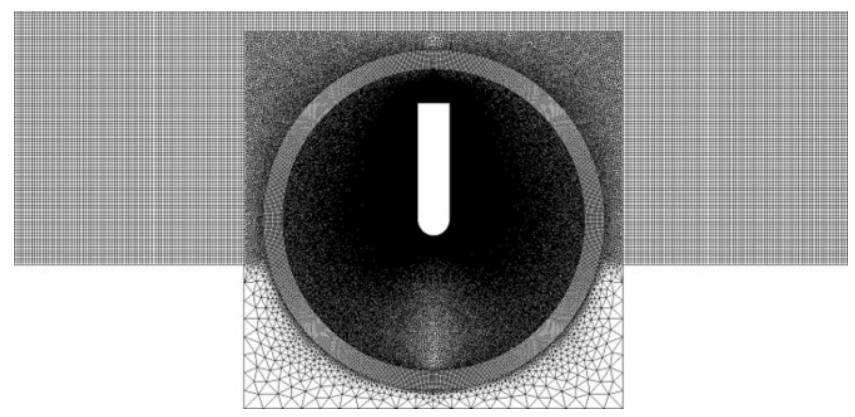

Fig. 2 Computational domain near the OWSC. The AMI interface is a circumference located in the middle of the ring meshing tool Gmsh. The physical dimensions of the computational domain are $16.77 \times 1.5 \times 0.646 \mathrm{~m}$ and correspond to the wave flume dimensions of the Ecole Centrale Marseille, where the experiments were carried out by Henry et al. (2014a). In our numerical setup, the center of the coordinates of the mesh coincides with the hinge, i.e., the axis of rotation, of the fortieth- scale OWSC featuring the dimensions of $0.875 \mathrm{~m} \times 0.31 \mathrm{~m} \times 0.646 \mathrm{~m}$. The center of gravity of the flap device is located at $0 \mathrm{~m}$, $0.1324 \mathrm{~m}$, and $0.323 \mathrm{~m}$. Its mass is $4.27 \mathrm{~kg}$, and its rotational inertia measured at the hinge is $0.1147 \mathrm{kgm}^{2}$.

The OWSC rotation (one degree of freedom) is computed through the use of OpenFOAM's dynamic mesh library (Jasak and Tukovic, 2006) in conjunction with OpenFOAM's Arbitrary Mesh Interface (AMI) library (Farrell and Maddison, 2011). To achieve this, the OWSC needs to be self-contained in a cylindershaped dynamic region of the computational mesh, which rotates around the flap hinge and interpolates information with the rest of the static computational domain through the AMI interface (see Fig. 2). The artificial mesh below the seabed that is necessary to accommodate the flap rotation, which also includes the structure fixing the flap to the sea bottom, is properly handled by a porosity term (see Fig. 4). This is an additional source term in the momentum equation that guarantees a zero velocity flux in that region. Finally, we rely on the third-party library ihFoam for the generation and absorption of incoming waves (Higuera et al., 2013).

The numerical conditions are set to match those of the experiments in which slamming was identified (see Henry et al., 2014a for further details). The mean water level is $0.305 \mathrm{~m}$ (the free surface is located at an abscissa of $0.2 \mathrm{~m}$ in the computational domain), and the regular waves based on the fifth-order Stokes theory (Higuera et al., 2013) are generated with a period of $1.9 \mathrm{~s}$ and a height of $0.1 \mathrm{~m}$ at the left boundary of the computational domain. No further calibration is done to match the experimental wave heights, which were carried out by driving a flap-type wavemaker using first-order theory (Ursell et al., 1960). Wave gauges (R1 to R7) are distributed along the numerical wave tank at locations $-10.978 \mathrm{~m},-7.022 \mathrm{~m},-6.842 \mathrm{~m},-6.482 \mathrm{~m},-6.125 \mathrm{~m}$, $-0.787 \mathrm{~m}$, and $0.785 \mathrm{~m}$, and a pressure gauge is placed at the seaward wall of the OWSC $(-0.04375 \mathrm{~m}, 0.21 \mathrm{~m}$, and $0.323 \mathrm{~m})$. Finally, in the simulations carried out in this work we utilize second-order discretization schemes for the spatial derivatives while the time integration is performed with a Euler backward scheme.

\section{NUMERICAL RESULTS}

\section{Convergence Tests}

The first battery of simulations is performed through the use of the incompressible Navier-Stokes solver. The aim of these preliminary tests is to verify the convergence of the numerical results and assess their agreement with respect to the available experimental data. The reference two-dimensional computational domain proposed in this work contains about 80,000 cells. The cell size of this reference mesh is reduced by a factor of two and four in each direction to generate two additional refined meshes accounting for approximately 200,000 and 900,000 cells, respectively.

The results corresponding to the OWSC angular velocity during three consecutive wave periods are presented in Fig. 3. Our numerical calculations are compared against the Queen's University Belfast (QUB) experiments performed in the Ecole Centrale Marseille's wave flume facility and the University College Dublin (UCD) results using Ansys Fluent (Henry et al., 2014a). It can be readily seen that our reference simulation (the red solid line) cannot capture well the dynamics of the flap, and thus a finer mesh is 


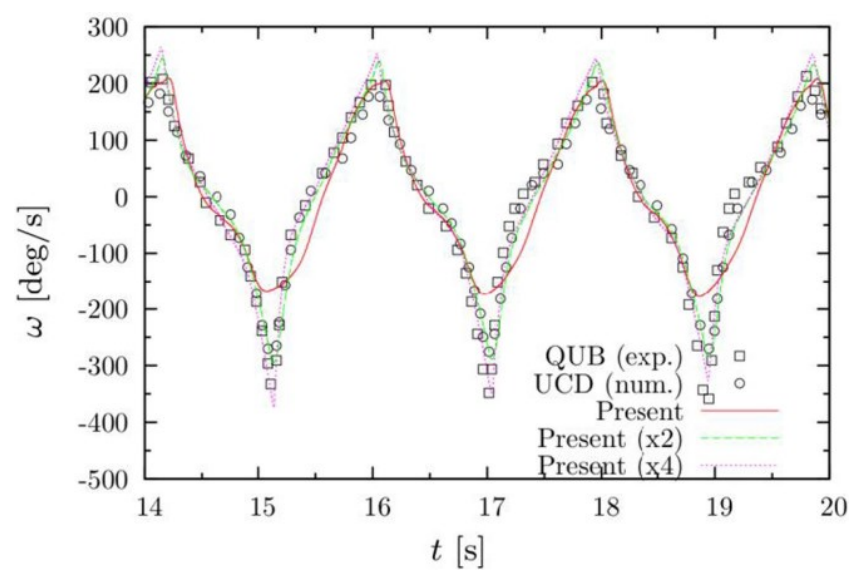

Fig. 3 OWSC angular velocity. Comparison of the present converged numerical results against previous data (Henry et al., 2014a)

required. The solution obtained with the finest mesh agrees quite well with the measured data and outperforms the UCD numerical results. Although it was not specified in Henry et al. (2014a), the UCD simulations probably used a coarser mesh or lower-order spatial discretization methods. Moreover, the difference between the reference mesh and the first refined mesh is less than the difference between the two refined meshes. Therefore, the use of one of the two refined meshes will provide reasonably well- converged results for this particular case.

Figure 4 shows the time history of the OWSC rotation angle, which is obtained by the integration of the flap angular velocity presented above. As expected from the results of Fig. 3, all the numerical results underestimate the measured negative rotation angles and overestimate the positive ones. As the mesh gets more refined, these differences tend to diminish for the negative angles and increase for the positive ones. In general, the OpenFOAM and Fluent incompressible solvers deliver similar results. However, the differences between the measured and simulated rotation angles are still not negligible and become even more evident at the leftmost and rightmost positions presented in the frame-byframe qualitative comparison shown in Fig. 5.

Henry et al. (2014a) pointed out that the discrepancy observed in Fig. 4 was associated with the drop in the water level, which was less significant in their simulations. In this work, we have

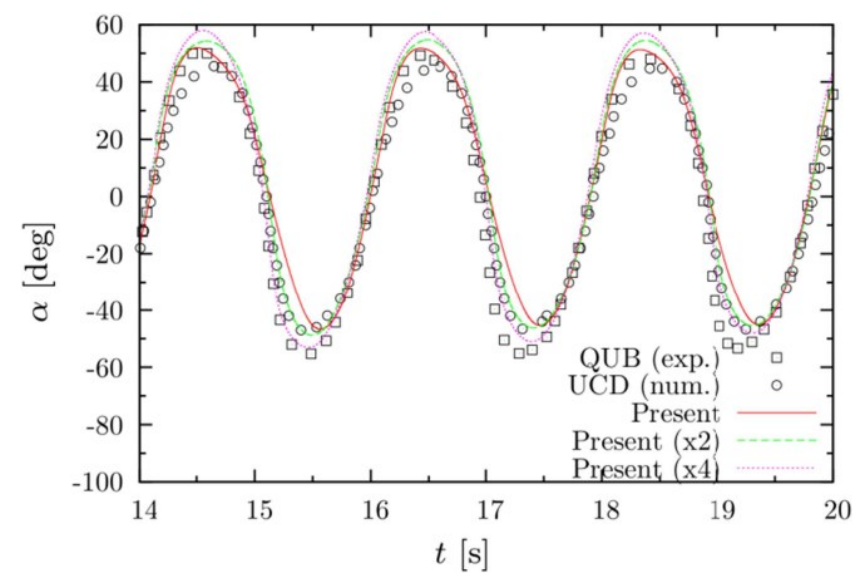

Fig. 4 OWSC rotation angle. Comparison of the present converged numerical results against previous data (Henry et al., 2014a)

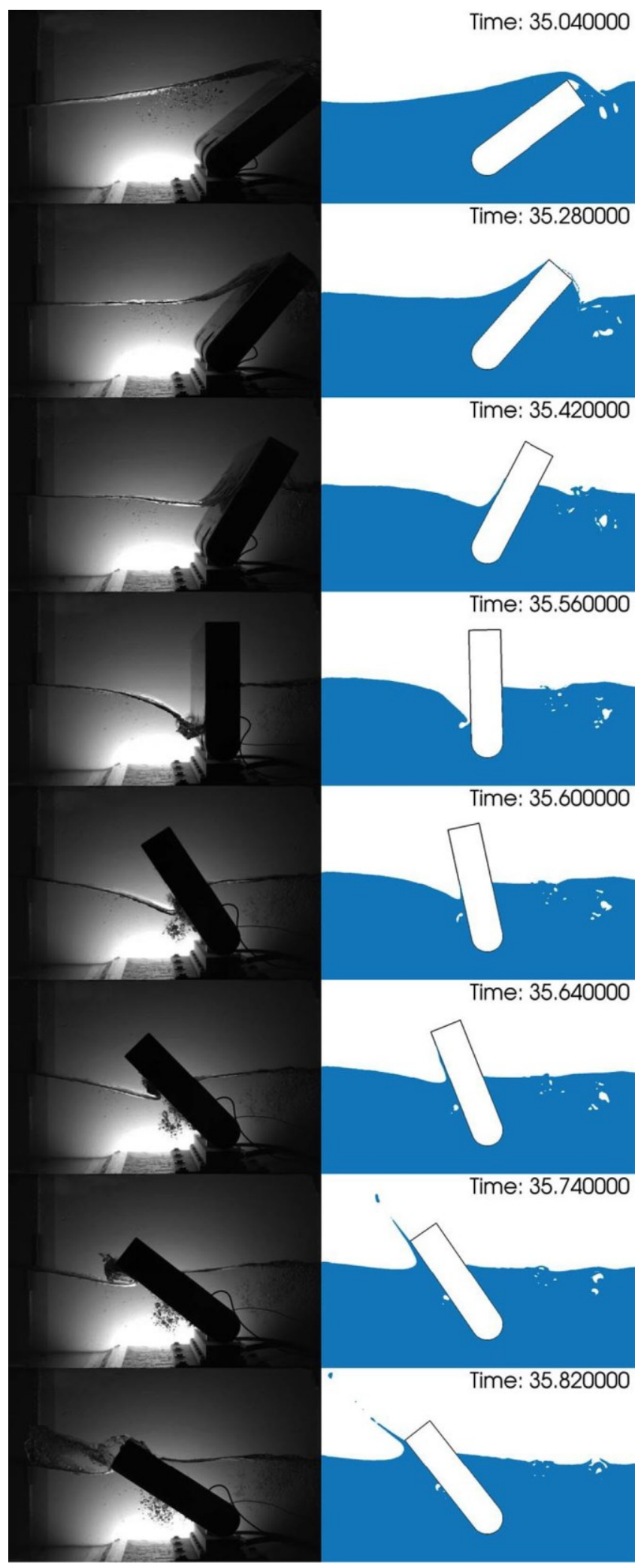

Fig. 5 Frame-by-frame comparison between experiments (left) and numerical simulations (right) carried out with OpenFOAM

conducted a series of converged simulations using different wave generation mechanisms and measured the wave elevation at gauge $\mathrm{R} 2$, which is

located at a distance of $5.178 \mathrm{~m}$ from the wavemaker (see Fig. 6). It can be readily seen that the waves generated with the 


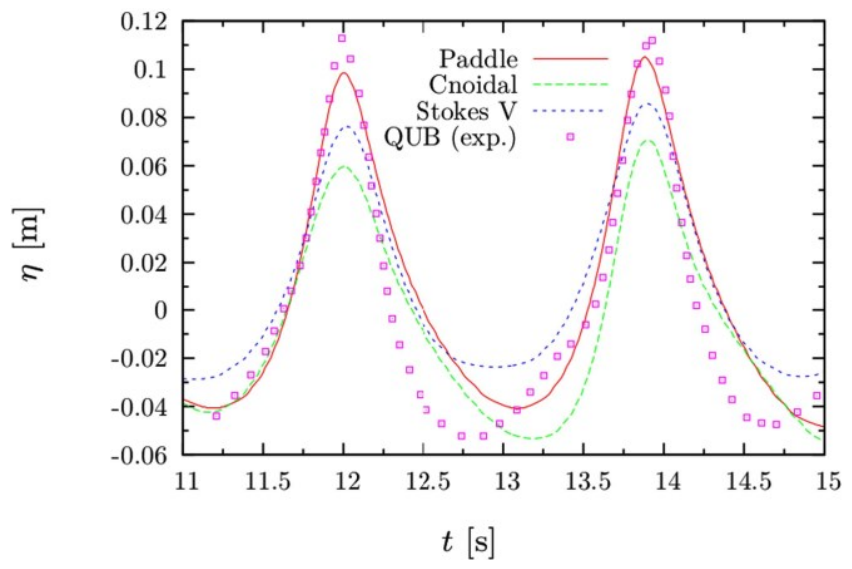

Fig. 6 Wave elevations at gauge R2. Comparison between numerical results and experimental data (Henry et al., 2014a)

fifth-order Stokes theory were slightly closer to the experiments compared to the ones provided by the cnoidal theory. However, these two solutions generated with a static wave generator boundary and a velocity profile (Higuera et al., 2013) systematically underestimated the experimental observations. Numerical wavemakers based on physical moving paddles may be more suitable to simulate experiments like this one. In a recent work, not shown here for the sake of conciseness, we carried out the same simulation using a moving flap-type numerical wavemaker (the red solid line in Fig. 6), but we were unable to replicate the experiment successfully.

Furthermore, the experiments carried out in the wave flume did not account for wave reflections, and the generated regular waves were largely influenced by this phenomenon (Wei et al., 2016). It is worth mentioning that the reflections on the walls of the tank, the wavemaker, and the OWSC will have an effect on the measured rotation angles and therefore slamming, especially during long-run experiments and computations. For example, Fig. 7 shows the wave elevations reported by gauges R6 and R7 situated at $0.786 \mathrm{~m}$ away from each side of the OWSC. It is clear that the wave conditions are constantly changing due to reflection, especially seaward of the OWSC (the red solid line), where there is a noticeable change in the negative elevations at around $38 \mathrm{~s}$. It is also worth mentioning that the static wave generator

boundary employed in this work reflects waves differently at a moving flap-type wavemaker.

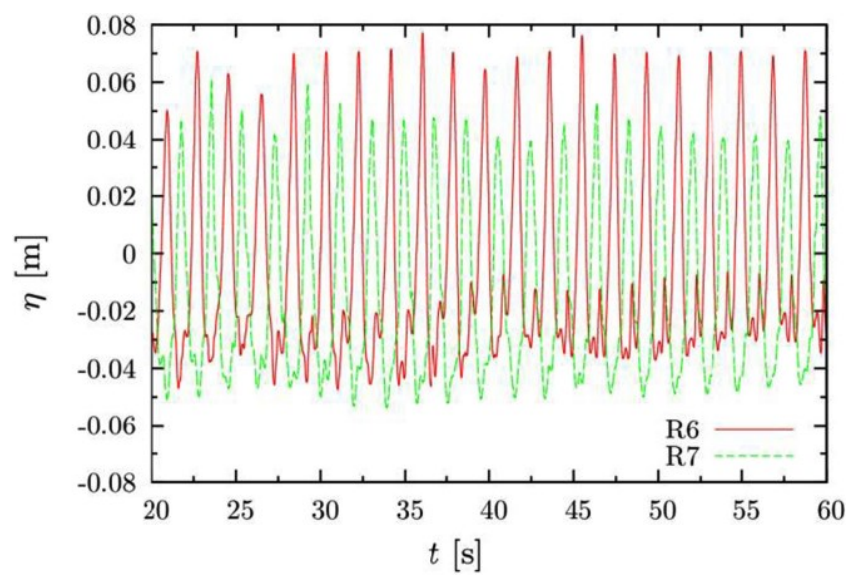

Fig. 7 Wave elevations at gauges R6 and R7

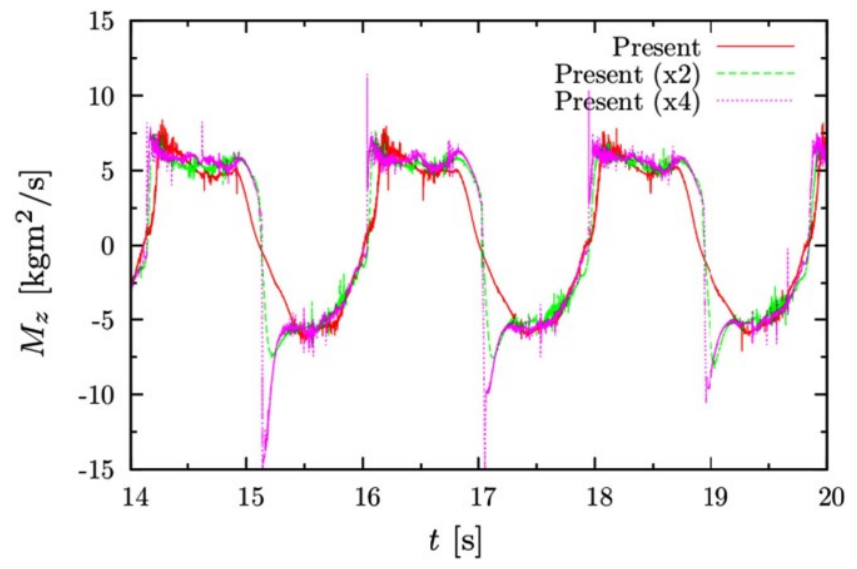

Fig. 8 OWSC torque measured at the hinge. Comparison between the present converged numerical results

It can be concluded from the figures presented above that (1) the dynamics and general behavior of the OWSC are well captured by an incompressible Navier-Stokes solver if the computational mesh is sufficiently refined; and (2) the discrepancies between the experiments and simulations can be attributed to the differences in the wave generation and reflection.

It is of great importance to evaluate accurately the forces acting on the OWSC as well. Figure 8 represents the evolution of the torque at the hinge as a function of the time. (There is no experimental or numerical data available for comparison.) The two refined solutions are relatively close compared to the reference solution. However, they diverge at the slamming moments (the negative values of the torque), where there seem to be convergence issues, i.e., unphysical oscillations. We believe that these oscillations are caused by the second-order spatial discretization schemes employed for the spatial derivatives.

Finally, Fig. 9 reports the recorded gauge pressure obtained by different numerical simulations. On the one hand, the UCD results present maximum pressure peaks of the order of $600 \mathrm{~Pa}$ above the ambient pressure. On the other hand, our results are slightly above that figure, with values reaching up to $900 \mathrm{~Pa}$. It is worth noting that these maximum pressure values remain very similar in the two refined meshes, which guarantee converged results for the pressure field. We have also identified very short pressure peaks taking place during the simulation (see Fig. 7), which we

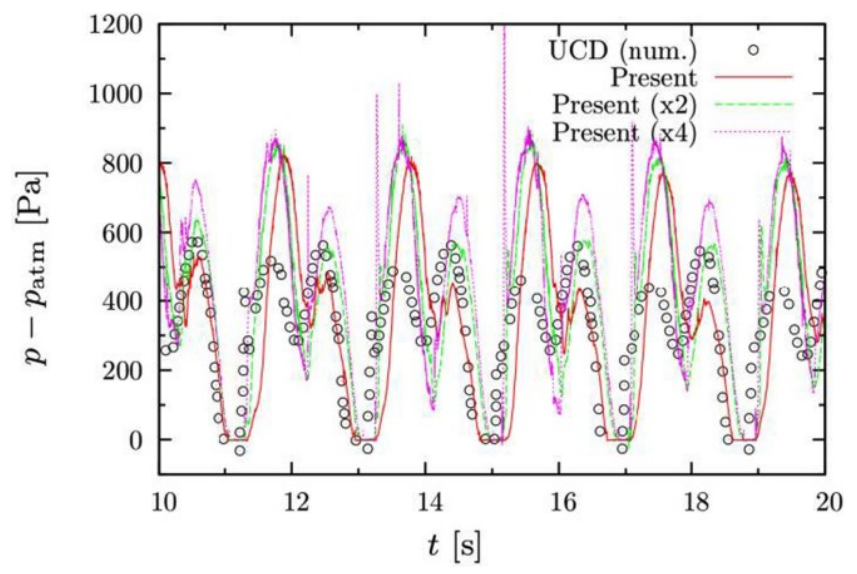

Fig. 9 Gauge pressure at the OWSC seaward wall. Comparison of the present converged numerical results against previous data (Henry et al., 2014a) 
believe are also caused by second-order spatially discretized terms on a fine unstructured mesh rather than by slamming phenomena. Similar pressure peaks not associated with slamming were also reported in previous computational works (Henry et al., 2014a).

\section{Scalability Results}

Fine meshes are a requirement to guarantee the convergence of the numerical results. The convergence of the pressure field is of great importance to detect the occurrence of slamming (see Fig. 9). Furthermore, long-run computations, which are about 60 seconds of physical time, are necessary to verify the repeatability of the wave conditions and to compare the simulations against the experiments. The complexity of the mesh, which accounts for the dynamic motion and AMI interpolation interfaces, has a considerable impact on the computational costs. Therefore, a new reference mesh composed of about 500,000 cells is proposed to carry out the long-run simulations in a reasonable amount of time while guaranteeing the convergence pressure results. Moreover, the size of this mesh lies between the sizes of the previous two refined meshes and permits the simulation to be over 60 seconds of physical time in less than 12 days through the use of a CFL number equal to 0.5 and 64 cores of the Neumann cluster installed at Manchester Metropolitan University (MMU).

We conducted scalability tests of the two-dimensional long-run simulations on two different architectures (see Fig. 10). The Neumann cluster installed at MMU is a small-size cluster featuring 12 computer nodes with Intel Xeon E5-2620 cores and an Infiniband QDR interconnect. ARCHER is a UK national Cray XC30 MPP supercomputer composed of 4,920 computer nodes with 12 Intel Ivy E5-2697 cores and a Cray Aries interconnect. Figure 8 shows that the Neumann scalability is somewhat compromised when communicating various nodes through the Infiniband interconnect. However, the same simulation runs fine on ARCHER with a fair scalability of 40 while using 64 cores (approximately 7,800 cells per core). For three-dimensional configurations such as the one presented by Wei et al. (2015), it will be necessary to use several million mesh cells, and this will require even a large number of nodes and HPC facilities. OpenFOAM can be used for HPC without associated extra license costs often present in commercial CFD software.

\section{Compressibility Results}

A new campaign of long-run numerical simulations was carried out through the use of both the incompressible and compressible Navier-Stokes solvers. The same mesh of about 500,000 cells

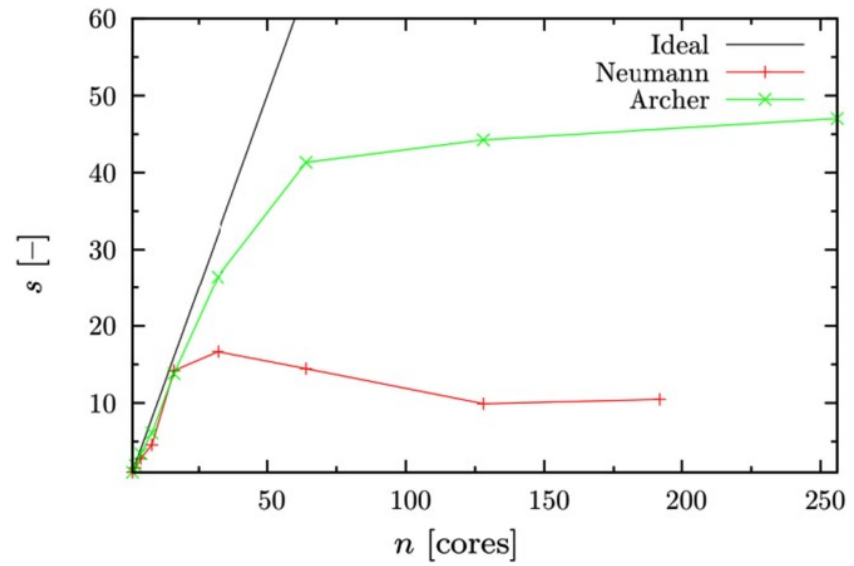

Fig. 10 OpenFOAM scalability for the OWSC two-dimensional simulations
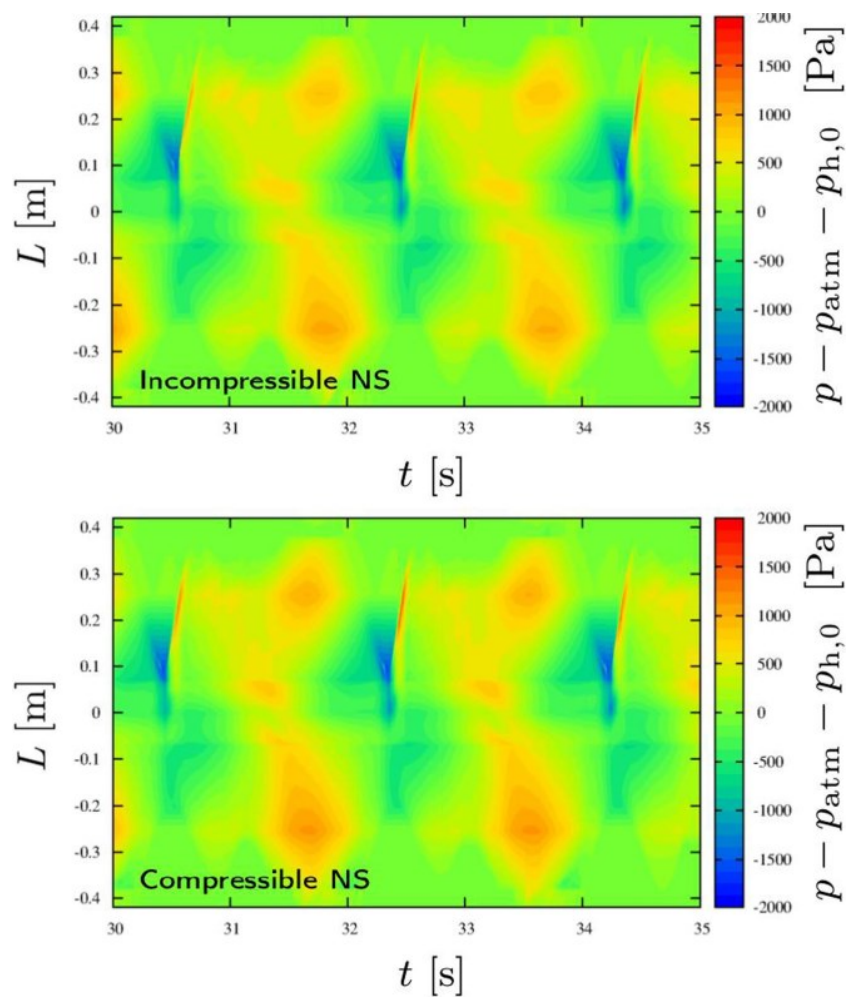

Fig. 11 Dynamic pressure history distribution along the surface of the OWSC for three consecutive slamming events

used in the scalability tests was employed, and we kept all the numerical parameters identical in order to ensure similar conditions for the two simulations. Figure 11 shows the dynamic pressure distribution along the OWSC surface as a function of the time in order to give a better illustration of the slamming phenomena. We would like to emphasize that the compressible simulations were more difficult to carry out as very strong numerical oscillations appeared, which led us to believe that they could be associated with slamming phenomena. After a careful examination of the iterative algorithms used in OpenFOAM, we conducted a new series of compressible simulations where this issue was removed. In Fig. 11, the vertical axis represents the perimeter; the positive values correspond to the seaward wall, while the negative ones belong to the opposite face. The lowest point of the OWSC, i.e., below the hinge, has a value of $0 \mathrm{~m}$. Both incompressible and compressible solvers can capture three consecutive slamming events, which can be identified as sharp pressure bands taking place approximately every $1.9 \mathrm{~s}$. Overall, our results agree qualitatively well with those presented by Wei et al. (2016). The similarity of the pressure ranges delivered by the incompressible and compressible solvers confirms that compressibility does not contribute to an increase of the maximum pressure peak values, as previously thought by Henry et al. (2014a) or from what can be derived in relation to the compressibility effects on the wave impacts (Bullock et al., 2007; Lugni et al., 2010). In fact, the maximum pressure values identified during slamming are directly associated with the velocity of the jet root that is formed as the OWSC pitches seaward, which is similar to a classic (incompressible) water- entry problem.

On the other hand, Fig. 12 shows in more detail the vertical pressure distribution along the OWSC seaward wall recorded during one slamming event (from $32.45 \mathrm{~s}$ to $33.15 \mathrm{~s}$ ). The purpose of this figure is to capture other compressibility effects that may be associated with the air entrapment observed in Fig. 5. It can 


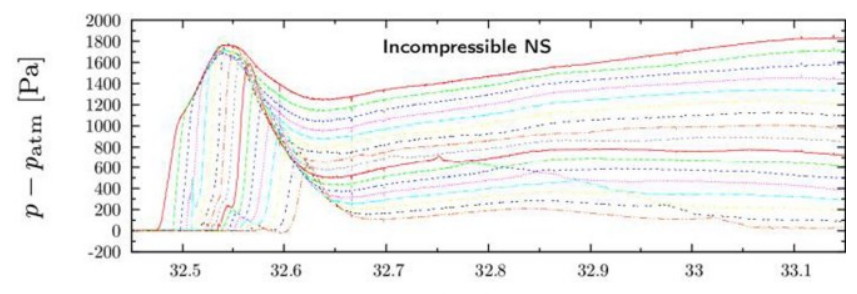

$t[\mathrm{~s}]$
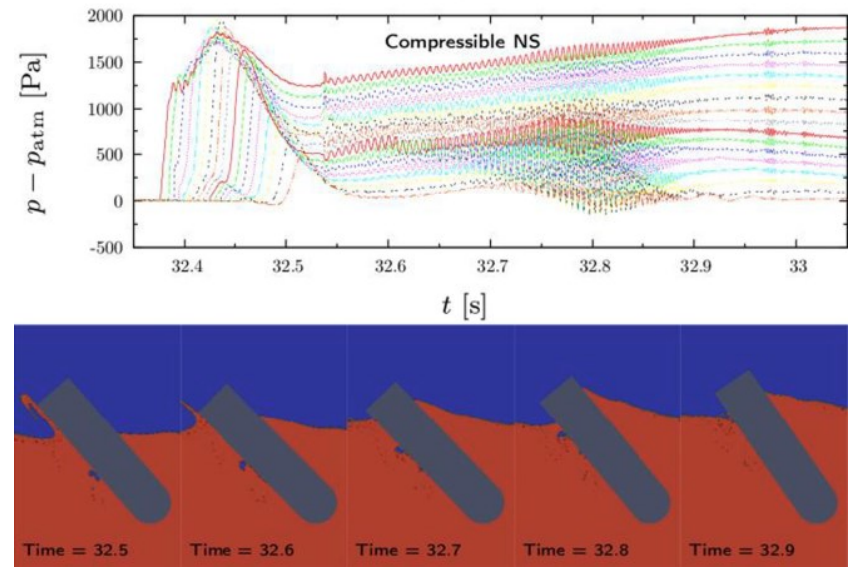

Fig. 12 Vertical pressure distribution along the OWSC seaward wall during a slamming event (top) and evolution of air bubbles during a slamming event (bottom)

be readily seen that the compressible solver is able to capture high-frequency (about $0.2 \mathrm{kHz}$ ), small-pressure oscillations along a region of the wall. Small, high-frequency pressure oscillations were also observed in a recent experiment conducted by Henry et al. (2015). In our numerical work, these oscillations correspond to the presence of a large air bubble near the seaward wall that is travelling upwards (see Fig. 12). When the bubble reaches the free surface, the oscillations disappear. It is also worth mentioning that while the lower sensors capture the largest pressure values, the higher ones are more sensitive to these small- pressure oscillations. In particular, the upper four sensors can be subject to negative values of the dynamic pressure, which can compromise the integrity of the OWSC structure and thus increase its operational and maintenance costs. Therefore, although compressibility does not contribute to further increasing the maximum impact loads of the OWSC, it causes high-frequency oscillations that can reach sub-atmospheric pressure values on the upper wall of the device.

\section{CONCLUSIONS}

The numerical modeling of an OWSC operating under slamming conditions characteristic of extreme sea states has been presented in this work. We performed two-dimensional numerical simulations with the aid of the open-source CFD library OpenFOAM using both incompressible and compressible NavierStokes solvers in order to assess whether compressibility effects play an important role during slamming events.

A preliminary study of convergence shows the importance of a fine mesh to capture well the dynamics and general behavior of the OWSC. Finer meshes, with their increasing demand on computational resources, become a requirement, and HPC simulations are necessary to perform long-run simulations or to deal with three-dimensional geometries in a reasonable amount of time. Therefore, the use of open-source software can be advantageous over commercial software due to the license costs of the HPC, which justifies our choice of using OpenFOAM.
The dynamics of the OWSC can be fairly predicted by an incompressible Navier-Stokes solver provided that the computational domain is correctly discretized and, in general, the simulation results are close to the available experimental data. The results obtained with a compressible Navier-Stokes solver have confirmed that (1) the compressibility effects do not increase the peak pressure values recorded during slamming, but that (2) the compressibility due to the entrapped air on the seaward wall produces high-frequency pressure oscillations, which can lead to subatmospheric values of the pressure.

Different campaigns of experiments (Henry et al., 2014a; Henry et al., 2015) have demonstrated the difficulty in reproducing the same experiment when slight changes are introduced in the wave tank. In this work, the wave generation based on a velocity profile can introduce errors, which can then be amplified in long-run simulations due to a different reflection system between the OWSC and a geometrically fixed wave generator boundary. Therefore, more investigations are necessary to close the gap between the experiments and the simulations. For instance, we recently conducted a study using a numerical dynamic wavemaker that can improve wave generation and reflection. Three-dimensional simulations will also be performed in the future for new campaigns of experiments to assess the importance of compressibility and the three-dimensional effects in the accurate prediction of loadings.

\section{ACKNOWLEDGEMENTS}

The authors acknowledge with gratitude the financial support from the Engineering and Physical Sciences Research Council (EPSRC) under the Software for the Future (SoFT) initiative and related research grants EP/K037889/1, EP/K038168/1, and EP/K038303/1.

\section{REFERENCES}

Bullock, GN, Obhrai, C, Peregrine, DH, and Bredmose, H (2007). "Violent Breaking Wave Impacts. Part 1: Results from Large Regular Wave Tests on Vertical and Sloping Walls," Coastal Eng, 54, 602-617. https://doi.org/10.1016/j.coastaleng.2006.12.002.

Farrell, PE, and Maddison, JR (2011). "Conservative Interpolation Between Volume Meshes by Local Galerkin Projection," Comput Methods Appl Mech Eng, 200, 89-100. https://doi.org/10.1016/j.cma.2010.07.015.

Henry, A, Abadie, T, Nicholson, J, McKinley, A, Kimmoun, O, and Dias, F (2015). "The Vertical Distribution and Evolution of Slam Pressure on an Oscillating Wave Surge Converter," Proc 34th Int Conf Ocean Offshore Arct Eng, St Johns, Canada, ASME, V001T01A034. https://doi.org/10.1115/OMAE2015-41290.

Henry, A, Kimmoun, O, Nicholson, J, Dupont, G, Wei, Y, and Dias, F (2014a). "A Two Dimensional Experimental Investigation of Slamming of an Oscillating Wave Surge Converter," Proc 24th Int Ocean Polar Eng Conf, Busan, Korea, ISOPE, 3, 296-305.

Henry, A, Rafiee, A, Schmitt, P, Dias, F, and Whittaker, TJT (2014b). "The Characteristics of Wave Impacts on an Oscillating Wave Surge Converter," J Ocean Wind Energy, ISOPE, 1, 101-110.

Higuera, P, Lara, JL, and Losada, IJ (2013). "Realistic Wave Generation and Active Wave Absorption for Navier-Stokes Models: Application to OpenFOAM," Coastal Eng, 71, 102-118. https://doi.org/10.1016/j.coastaleng.2012.07.002. 
Jasak, H (1996). Error Analysis and Estimation for the Finite Volume Method with Applications to Fluid Flows, PhD Thesis, University of London, UK.

Jasak, $\mathrm{H}$, and Tukovic, $\mathrm{Z}$ (2006). "Automatic Mesh Motion for the Unstructured Finite Volume Method," Trans FAMENA, 30, $1-20$.

Kay, J (2014). UK Storms Destroy Railway Line and Leave Thousands without Power, Retrieved from http://www.bbc.co.uk/ news/uk-26042990.

Lugni, C, Brocchini, M, and Faltinsen, OM (2010). "Evolution of the Air Cavity during a Depressurized Wave Impact. II. The Dynamic Field," Phys Fluids, 22, 056102. https://doi.org/10.1063/1.3409491.

Ma, ZH, Causon, DM, Qian, L, Mingham, CG, Gu, HB, and Martínez Ferrer, P (2014). "A Compressible Multiphase Flow Model for Violent Aerated Wave Impact Problems," Proc $R$ Soc London Ser A, 470, 2014.0542. https://doi.org/10.1098/rspa.2014.0542.

Martínez Ferrer, PJ, Causon, DM, Qian, L, Mingham, CG, and $\mathrm{Ma}, \mathrm{ZH}$ (2016). "A Multi-region Coupling Scheme for Compressible and Incompressible Flow Solvers for Two-phase Flow in a Numerical Wave Tank," Comput Fluids, 125, 116-129. https://doi.org/10.1016/j.compfluid.2015.11.005.

Rusche, H (2002). Computational Fluid Dynamics of Dispersed
Two-phase Flows at High Phase Fractions, PhD Thesis, University of London, UK.

Ursell, F, Dean, RG, and Yu, YS (1960). "Forced Small-amplitude Water Waves: A Comparison of Theory and Experiment," J Fluid Mech, 7(1), 33-52. https://doi.org/10.1017/S0022112060000037.

Wei, Y, Abadie, T, Henry, A, and Dias, F (2016). "Wave Interaction with an Oscillating Wave Surge Converter, Part II: Slamming," Ocean Eng, 113, 313-334.

https://doi.org/10.1016/j.oceaneng.2015.12.041.

Wei, Y, Rafiee, A, Henry, A, and Dias, F (2015). "Wave Interaction with an Oscillating Wave Surge Converter, Part I: Viscous Effects," Ocean Eng, 104, 185-203.

https://doi.org/10.1016/j.oceaneng.2015.05.002.

Weller, HG (2002). Derivation, Modelling and Solution of the Conditionally Averaged Two-phase Flow Equations, Technical Report TR/HGW/02, Nabla Ltd.

Whittaker, T, and Folley, M (2012). "Nearshore Oscillating Wave Surge Converters and the Development of Oyster," Philos Trans $R$ Soc London Ser A, 370, 345-364. https://doi.org/10.1098/rsta.2011.0152.

Xu, GD, Duan, WY, and Wu, GX (2010). "Simulation of Water Entry of a Wedge through Free Fall in Three Degrees of Freedom," Proc R Soc London Ser A, 466, 2219-2239. https://doi.org/10.1098/rspa.2009.0614. 